\title{
Signatures of chaos and non-integrability in two-dimensional gravity with dynamical boundary
}

\author{
Maxim Fitkevich ${ }^{1,2, \star}$ \\ ${ }^{1}$ Institute for Nuclear Research of the Russian Academy of Sciences, 60th October Anniversary Prospect, \\ 7a, 117312 Moscow, Russia \\ ${ }^{2}$ Moscow Institute of Physics and Technology, Institutskii per. 9, Dolgoprudny 141700, Moscow Region, \\ Russia
}

\begin{abstract}
We propose a model of two-dimensional dilaton gravity with a boundary. In the bulk our model coincides with the classically integrable CGHS model; the dynamical boundary cuts off the CGHS strong-coupling region. As a result, classical dynamics in our model reminds that in the spherically-symmetric gravity: wave packets of matter fields either reflect from the boundary or form black holes. We find large integrable sector of multisoliton solutions in this model. At the same time, we argue that the model is globally non-integrable because solutions at the verge of black hole formation display chaotic properties.
\end{abstract}

\section{Introduction}

About half a century ago Hawking discovered [1] that black holes thermally evaporate exhibiting an apparent loss of quantum coherence. This so-called information paradox contradicts to the principles of quantum theory where a pure initial quantum state $\left|\psi_{i n}\right\rangle$ cannot evolve into a one. To resolve this puzzle, theorists try to keep track of quantum information within the black hole interior. The current understanding of the information paradox relies on the AdS/CFT argument [2] which relates black hole formation to a thermalization of a quantum state in a dual CFT. The latter process, albeit unitary, dynamically hides quantum coherence in the an approximately thermal final state. This suggests that investigation of dynamical properties of black hole formation may be helpful for resolving the information paradox.

Recently, it was argued [3],[4] that particle collisions near the black hole horizon display chaotic behaviour: their final states are very sensitive to the initial conditions. This dynamical chaos may be significant for the apparent loss of quantum coherence during black hole formation and evaporation.

In this note based on the forthcoming publication [5] we propose a toy model of dilaton gravity which has several desired properties. On one hand, the model possesses a large sector of exact solutions which describe either gravitational scattering or collapse of matter. On the other hand, the solutions in our model are extremely sensitive to the initial conditions at the verge of black hole formation implying that the model is globally non-integrable.

^e-mail: fitkevich@phystech.edu 


\section{The model}

The basis for construction of our model is a two-dimensional CGHS gravity [6]. In the CGHS model the metric $g_{\mu \nu}$ is coupled to a dilaton field $\phi$ and a massless free scalar field $f$ representing matter, the action is ${ }^{1}$

$$
S_{C G H S}=\int d^{2} x \sqrt{-g}\left[e^{-2 \phi}\left(R+4(\nabla \phi)^{2}+4 \lambda^{2}\right)-\frac{1}{2}(\nabla f)^{2}\right] .
$$

Here $R$ is the Ricci scalar, $\lambda^{2}$ is the cosmological constant. The model (1) is exactly solvable at the classical level. The metric and the dilaton can be explicitly obtained from constraints, which leaves the field $f$ as the only dynamical degree of freedom.

Unfortunately, the CGHS model itself cannot be used as a consistent model at the semiclassical level. In Minkowski vacuum

$$
g_{\mu \nu}=\eta_{\mu \nu}, \quad \phi=-\lambda x^{1},
$$

the dilaton field tends to infinity at $x^{1} \rightarrow-\infty$ implying infinitely large gravitational coupling constant $e^{\phi}$ in this region, see Fig. 1a and cf. Eq (1). This makes the model strongly coupled at large negative $x^{1}$ and restricts applicability of the semiclassical calculations.

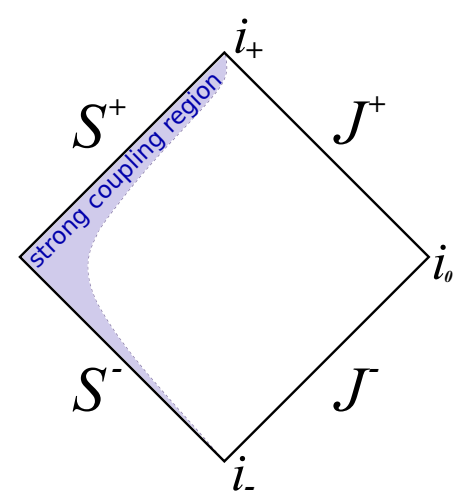

(a)

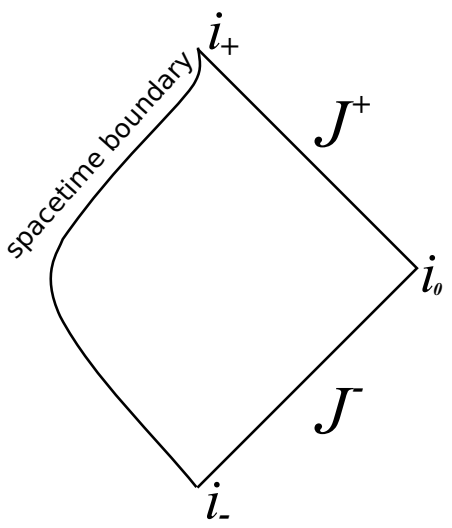

(b)

Figure 1. Penrose diagrams for the Minkowski spacetime in the (a) CGHS model and (b) model with a boundary.

In order to avoid this difficulty we add a dynamical boundary at $\phi=\phi_{0}$ cutting off the strong coupling region at large $\phi$. Namely, we restrict integration in Eq. (1) to the manifold $\mathcal{M}$ where $\phi<\phi_{0}$ and add a Gibbons-Hawking boundary term,

$$
S=\left.S_{C G H S}\right|_{\mathcal{M}}+2 \oint_{\partial \mathcal{M}} d \sigma \sqrt{|h|} e^{-2 \phi}(K+2 \lambda),
$$

where $K=g^{\mu \nu} \nabla_{\mu} n_{v}$ is the extrinsic curvature of the boundary, $n^{v}$ is the outer normal. A new parameter $e^{\phi_{0}}$ can be regarded as a gravitational coupling constant in our model. Previously a similar model with dynamical mirror has been proposed in [8]. Unlike the authors of [8] we analyse integrable properties of this model at the classical level rather than trying to quantize it.

\footnotetext{
[7].

${ }^{1}$ The first term in Eq. (1) can be obtained by spherical reduction of the d-dimensional Einstein gravity in the limit $d \rightarrow+\infty$
} 


\section{Field equations}

The starting point in simplifying the field equations is to find a general solution in the bulk. We work in conformal coordinates $(u, v)$, where the interval has the form $d s^{2}=-e^{2 \rho} d v d u$. Besides, we choose the frame with $\rho=\phi$. Field equation for $f(u, v)$ in these coordinates can be explicitly solved,

$$
\square f \equiv-4 e^{-2 \rho} \partial_{v} \partial_{u} f=0 \quad \Rightarrow \quad f(v, u)=f_{\text {out }}(u)+f_{\text {in }}(v) .
$$

Expressions for the other fields follow from the constraints,

$$
e^{-2 \rho} \equiv e^{-2 \phi}=-\lambda^{2} v u+g(v)+h(u),
$$

where

$$
g(v)=\frac{1}{2} \int_{0}^{v} d v^{\prime} \int_{v^{\prime}}^{\infty} d v^{\prime \prime} f_{v}^{2}\left(v^{\prime \prime}\right), \quad h(u)=-\frac{1}{2} \int_{-\infty}^{u} d u^{\prime} \int_{-\infty}^{u^{\prime}} d u^{\prime \prime} f_{u}^{2}\left(u^{\prime \prime}\right) .
$$

Equations (4)-(6) give the general solution in the bulk.

Now, we impose conditions at dynamical boundary $\phi=\phi_{0}$. Varying Eq. (3) with respect to the boundary values of $g_{\mu \nu}$ and $f$, we obtain,

$$
\left.n^{\mu} \nabla_{\mu} f\right|_{\phi=\phi_{0}}=0,\left.\quad \quad n^{\mu} \nabla_{\mu} \phi\right|_{\phi=\phi_{0}}=\lambda
$$

The first of these equations implies that the energy flux through the boundary equals zero. The second condition guarantees that the flat spacetime (2) is a solution. Integrating the first of Eqs. (7) we obtain a diffeomorphism between in- and out-going wave packets,

$$
f_{\text {out }}(U(v))=f_{\text {in }}(v)
$$

where $u=U(v)$ is the trajectory of the boundary in the $(u, v)$ frame: $\phi(U(v), v)=\phi_{0}$.

Using Eqs. (5), one derives an equation for $U(v)$ from the conditions (7),

$$
U_{v}=\frac{e^{2 \phi_{0}}}{\lambda^{2}}\left(g_{v}-\lambda^{2} U\right)^{2},
$$

where $\partial_{v} g$ can be derived from Eq. (6). Introducing auxiliary function $\psi(v)$, satisfying

$$
e^{-2 \phi_{0}} \frac{\psi_{v}}{\psi}=\partial_{v} g-\lambda^{2} U
$$

we further simplify this equation,

$$
\psi_{v v}(v)=\mathcal{T}(v) \psi(v),
$$

where $\mathcal{T}=e^{2 \phi_{0}} g_{v v}$ depends on the incoming wave packet. At $\mathcal{T}=0$ one obtains the Minkowski solution

$$
\psi(v)=\lambda v \quad \text { and } \quad U(v)=-\frac{e^{-2 \phi_{0}}}{\lambda^{2} v},
$$

which is equivalent to Eq. (2) after proper change of coordinates

$$
-\lambda u=e^{-\lambda \bar{u}}, \quad \lambda v=e^{\lambda \bar{v}}, \quad \text { where } \quad(\bar{v}, \bar{u})=\left(x^{0}+x^{1}, x^{0}-x^{1}\right) .
$$

In what follows we use $(\bar{v}, \bar{u})$ as asymptotically flat coordinates. 


\section{Integrable sector}

Exact solutions in our model are obtained in the following way. Suppose $\psi(v)$ in Eq. (11) is analytic everywhere except for $N$ isolated singularities $v_{i}$, where $\psi \propto\left(v-v_{i}\right)^{-s_{i}}$. In the vicinity of $v_{i}$ Eq. (11) gives,

$$
\mathcal{T}=\frac{\psi_{v v}}{\psi} \sim \frac{s_{i}\left(s_{i}+1\right)}{\left(v-v_{i}\right)^{2}} .
$$

Thus, function $\mathcal{T}(v)$ has only the poles of rank 2 , and we write,

$$
\mathcal{T}(v)=\sum_{i=1}^{N}\left(\frac{s_{i}\left(s_{i}+1\right)}{\left(v-v_{i}\right)^{2}}+\frac{\mathcal{T}_{i}}{v-v_{i}}\right), \quad \mathcal{T} \leq 0 \quad \text { at } \quad v>0 .
$$

In what follows we consider for simplicity only the singularities of rank $s_{i}=1$. Substituting Laurent series,

$$
\begin{gathered}
\psi \sim \frac{\psi_{-1}^{i}}{v-v_{i}}+\psi_{0}^{i}+\psi_{1}^{i}\left(v-v_{i}\right)+\psi_{2}^{i}\left(v-v_{i}\right)^{2}+\ldots, \\
\mathcal{T} \sim \frac{2}{\left(v-v_{i}\right)^{2}}+\frac{\mathcal{T}_{i}}{v-v_{i}}+\mathcal{T}_{0}^{i}+\mathcal{T}_{1}^{i}\left(v-v_{i}\right)+\ldots \quad \text { at } \quad v \rightarrow v_{i}
\end{gathered}
$$

into Eq. (11), one obtains an infinite chain of equations for the Laurent coefficients,

$$
\begin{aligned}
& 2=2 \\
& \psi_{0}=-\frac{1}{2} \mathcal{T}_{i} \psi_{-1} \\
& \psi_{1}=\left(-\frac{1}{2} \mathcal{T}_{0}^{i}+\frac{1}{4} \mathcal{T}_{i}^{2}\right) \psi_{-1}, \\
& \mathcal{T}_{1}^{i} \psi_{-1}+\mathcal{T}_{i} \psi_{1}+\mathcal{T}_{0}^{i} \psi_{0}=0 \\
& \psi_{2}=\ldots
\end{aligned}
$$

We note that two equations in the set (18) are special since $\psi_{-1}$ is not present in Eq. (18a), as well as $\psi_{2}$ in Eq. (18d). Thus, $\psi_{-1}$ and $\psi_{2}$ are free parameters of the solution, while Eqs. (18a) and (18d) are constraints on the function $\mathcal{T}(v)$. Using Eqs. (18b), (18c), we rewrite Eq. (18d) in the form,

$$
\mathcal{T}_{1}^{i}=\mathcal{T}_{0}^{i} \mathcal{T}_{i}-\frac{1}{4} \mathcal{T}_{i}^{3}
$$

The other constraints are obtained from a requirement for solutions to be localized, i.e. $\mathcal{T}(v)=o\left(v^{-2}\right)$ at $v \rightarrow+\infty$. This gives two conditions,

$$
\sum_{i=1}^{N} \mathcal{T}_{i}=0, \quad \quad \quad \sum_{i=1}^{N}\left(2+v_{i} \mathcal{T}_{i}\right)=0
$$

Solving system of algebraic equations (19), (20) one obtains coefficients $\mathcal{T}_{i}$. i.e. the exact solution for $\mathcal{T}(v)$ in the form $(15)$.

Now, consider the solution with two singularities, $s_{1}=s_{2}=1$. Reality of $\mathcal{T}(v)$ gives $v_{1}=v_{2}^{*}=$ $v_{0}+i T$. Solving the conditions (20), one obtains,

$$
\mathcal{T}=-\frac{8 T^{2}}{\left[\left(v-v_{0}\right)^{2}+T^{2}\right]^{2}} .
$$


Then Eqs. (18) give the Laurent coefficients of $\psi(v)$, and we find,

$$
\psi=1+\frac{T^{2}-3 v_{0}^{2}}{8 v_{0} T^{2}} v-\frac{\frac{4}{3} T^{2}}{\left(v-v_{0}\right)^{2}+T^{2}}\left[1+\frac{T^{2}-3 v_{0}^{2}}{8 v_{0} T^{2}}\left(3 v-2 v_{0}\right)\right]
$$

It is straightforward to implement the procedure of this Section for finding exact solutions with $N$ singularities of different ranks.

\section{Reflecting and collapsing solutions}

We restrict our analysis to the localized wave packets $\mathcal{T} \propto f_{v}^{2}(v)$. In this case $\psi(v) \sim C v$ at $v \rightarrow+\infty$, see Eq. (11). We will see that the coefficient $C$ determines regimes of behaviour and plays a role of a critical parameter; in the case (22) we obtain $C=\frac{T^{2}-3 v_{0}^{2}}{8 v_{0} T^{2}}$. Solution (22) at different values of $C$ is shown in Fig. 2.

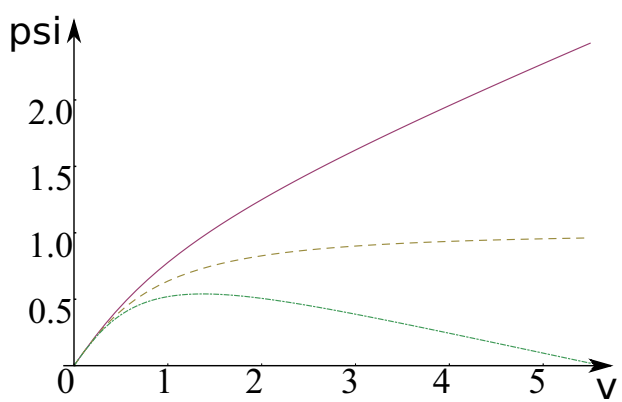

(a)

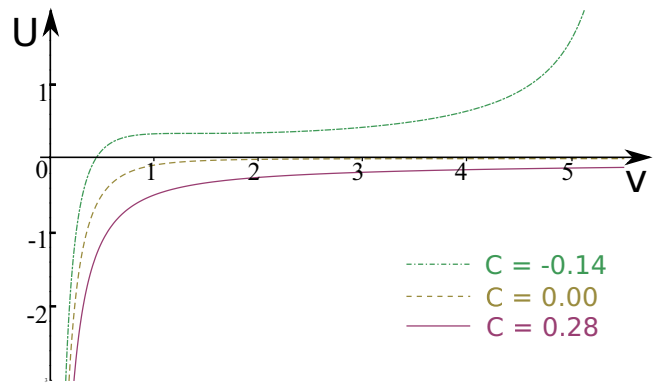

(b)

Figure 2. Plots of (a) $\psi(v)$ and (b) $U(v)$ for the exact solution (22) at $T=1$ and different values of the parameter $C=C\left(v_{0}\right)$.

At $C>0$ one shows that $U(v) \sim-\frac{e^{-2 \phi_{0}}}{\lambda^{2} v}+o\left(v^{-1}\right)$ at $v \rightarrow+\infty$, see Eq. (10). This coincides asymptotically with the Minkowski solution (12); thus $C>0$ corresponds to total reflection of the matter from the boundary.

Solutions with $C<0$ describe collapse. Indeed, in this case the function $\psi(v)$ has a maximum $\psi_{v}=0$ which corresponds to the singularity $d U / d v=0$ where the transformation $u=U(v)$ cannot be inverted. This is the singularity point of the outgoing wave packet $f_{\text {out }}(u)$ in Eq. (8). Appearance of this singularity signals that the black hole is formed in the final state of the process.

In the critical case $C=0$ the boundary becomes asymptotically null $U(v)=o\left(v^{-1}\right)$ at $v \rightarrow+\infty$. One can show that energy difference between the incident and reflected wave packets in this case is equal to $M_{c r}=2 \lambda e^{-2 \phi_{0}}$ and corresponding bulk solution at $i^{+}$describes CGHS black hole with mass $M_{c r}$. The critical solutions with $C=0$ separate the families of the reflecting and collapsing solutions. One can show that solutions at small negative $C$ form a black hole with minimal mass $M_{c r}$. On the other hand, the solutions with small positive $C$ describe total reflection. 


\section{Nonintegrability in the critical regime}

The CGHS model in the bulk is exactly solvable and has an infinite number of conserved quantities in the in- and out-sectors. In fact, any functionals of $f_{\text {in }}$ and $f_{\text {out }}$, e.g.

$$
I_{k}^{i n}=\int d v^{\prime}\left(\partial_{v} f_{\text {in }}\left(v^{\prime}\right)\right)^{k}, \quad \quad I_{k}^{\text {out }}=\int d u^{\prime}\left(\partial_{u} f_{\text {out }}\left(u^{\prime}\right)\right)^{k}
$$

can be regarded as such conserved quantities. The question is whether these quantities can be smoothly joined at the boundary to represent the integrals in our model? This would require the map $\left\{I_{i}^{\text {in }}\right\} \mapsto\left\{I_{j}^{\text {out }}\right\}$ in Eq. (8) to be smooth. We are going to argue, however, that this is not the case: the map $\left\{I_{i}^{\text {in }}\right\} \mapsto\left\{I_{j}^{\text {out }}\right\}$ is essentially singular in the vicinity of the near-critical solutions.

To understand the singular behaviour in the critical regime, consider the trajectory of the boundary in asymptotic coordinates (13),

$$
\bar{u}(v)=\frac{2 \phi_{0}}{\lambda}-\frac{1}{\lambda} \ln \left(\frac{\psi_{v}(v)}{\lambda \psi(v)}-\frac{2 g_{v}(v)}{M_{c r}}\right), \quad \lambda v=e^{\lambda \bar{v}},
$$

where Eq. (10) was used. The typical trajectory in the near-critical regime is shown in Fig. 3a. It has a large "plateau" part $v_{1} \leq v \leq v_{2}$ which size grows to infinity as $C \rightarrow 0$. Besides, one can argue that the trajectory $\bar{u}(\bar{v})$ within the "plateau" becomes null in the limit $C \rightarrow 0$. Thus, the part of the initial wave packet $f_{i n}(v)$ which gets reflected from the "plateau" gets blue shifted and shrinks into a narrow pulse in the outgoing packet. The latter part of the outgoing wave packet shrinks to a delta-peak at $C \rightarrow 0$; in what follows we call it "thunderpop".

Let us describe reflection of wave packets from the boundary in the limit $C \rightarrow 0$. At large $v$ we expect,

$$
\psi(v)=1+C v+\psi^{(1)}+\psi^{(2)}+\ldots,
$$

where $\psi^{(2)} \ll \psi^{(1)} \ll 1$ at $v \rightarrow+\infty$. Besides, we assume that $C v$ and $\psi^{(1)}$ are of the same order. Solving Eq. (11) perturbatively and recalling that $g_{v v} \propto v^{-2}$ is small, we obtain,

$$
\begin{aligned}
\psi^{(1)}(v)= & e^{2 \phi_{0}} \int_{v}^{\infty} d v^{\prime} \int_{v^{\prime}}^{\infty} d v^{\prime \prime} g_{v v}\left(v^{\prime \prime}\right)=e^{2 \phi_{0}}(g(v)-g(\infty)), \\
& \psi_{v}^{(2)}(v)=-C \lambda e^{2 \phi_{0}}\left(g(v)-g(\infty)-v g_{v}(v)\right)+ \\
+ & e^{4 \phi_{0}}\left((g(v)-g(\infty)) g_{v}(v)+\int_{v}^{\infty} d v^{\prime}\left(g_{v}\left(v^{\prime}\right)\right)^{2}\right) .
\end{aligned}
$$

Next, we calculate the outgoing wave packet using Eq. (8),

$$
f_{\bar{u}}^{\text {out }}(\bar{u}(v))=\lambda^{3} e^{2 \phi_{0}} f_{v}(v)\left(\frac{\psi(v)}{\psi_{v}(v)}\right)^{2} U(v) .
$$

Substituting the perturbative solution (25), (26), we find,

$$
\begin{gathered}
f_{\bar{u}}^{\text {out }}(\bar{u}(v)) \approx \frac{C f_{v}(v)}{\left(C+e^{2 \phi_{0}} \frac{g_{v}(v)}{\lambda}\right)^{2}}, \\
\bar{u}(v) \approx \frac{2 \phi_{0}}{\lambda}-\frac{1}{\lambda} \ln C+\text { corrections } .
\end{gathered}
$$

Thus the boundary is indeed almost null, $\bar{u} \approx$ const, in the large "plateau" region $C v \sim \psi^{(1)}$. 
To illustrate instability of the critical solution, we slightly change the initial wave packet by modulating its tail,

$$
\mathcal{T}=\mathcal{T}_{\text {sol }} \cos ^{2}(\omega \ln (\lambda v)), \quad \text { at } \quad v>v_{1},
$$

where $v_{1} \gg v_{0}$ and $\mathcal{T}_{\text {sol }}$ is given by Eq. (21). In the limit $\omega \rightarrow 0$ the original solution is restored. Let us check whether the integrals $I_{k}^{\text {out }}$ are smooth functions of $\omega$ and $C$ parametrizing the solutions.

For simplicity, we consider the third integral

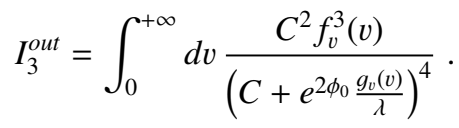

Substituting the asymptotics of (30) we obtain a combination of power-law and periodic functions in the integrand of Eq. (31). Rescaling the integration variable $v \mapsto v e^{2 \pi n / \omega}$, one proves an identity

$$
I_{3}\left(\omega, e^{6 \pi n / \omega} C\right)=e^{-2 \pi n / \omega} I_{3}(\omega, C) .
$$

This functional equation has a solution

$$
I_{3}(\omega, \ln C)=\frac{J(\omega \ln C)}{C^{\frac{1}{3}}},
$$

where $J(x)$ is periodic $J(x)=J(x+6 \pi n)$.

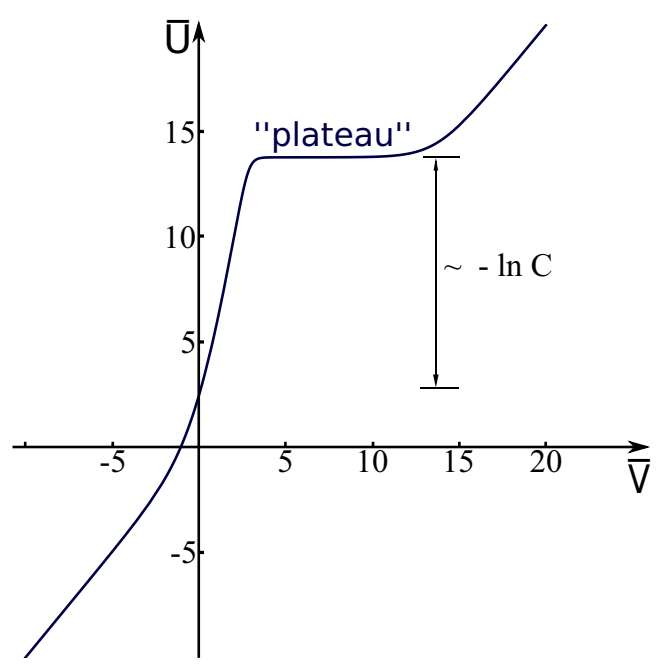

(a)

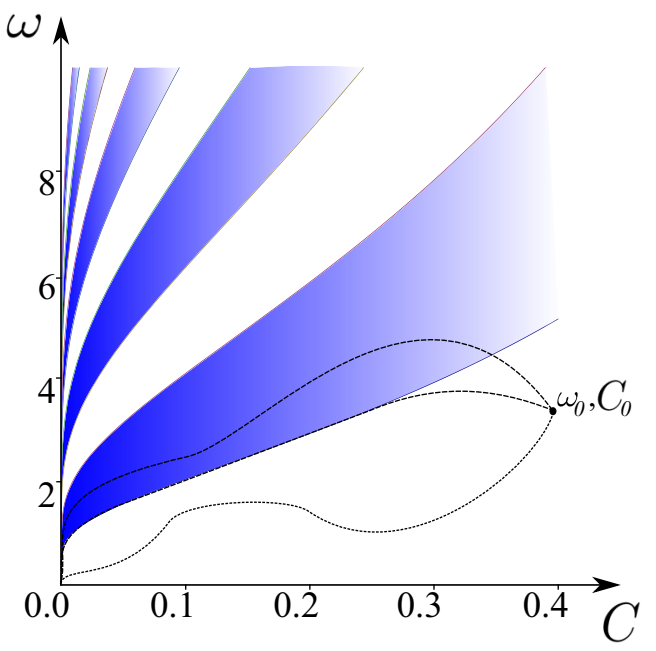

(b)

Figure 3. (a) Apparent boundary trajectory for the solution (22) at $T=1$ and $C=10^{-6}$. (b) Function $I_{3}$ on the plane $(C, \omega)$. White and blue regions correspond to $I_{3}>0$ and $I_{3}<0$, respectively. At the boundaries of these regions $I_{3}=0$.

Given representation (33), one immediately concludes that the limit of $I_{3}(\omega, C)$ at $\omega \rightarrow 0$ and $C \rightarrow 0$ essentially depends on the method of evaluating: one can make it equal to any real number by choosing the limiting path $\omega(C)$, see Fig. $3 b$. In other words, $I_{3}$ has an essential singularity at $\omega \rightarrow 0$, $C \rightarrow 0$ and the internal structure of the thunderpop is sensitive to small variations of $f_{i n}$. 
To summarize, the structure of the final pulse is unstable under the small variations of initial data. Despite our intuition the final pulse could have a non-trivial peak structure, which depends on values of $\omega$ and $C$. If we choose $\omega \simeq \frac{6 \pi n}{\ln C}$, the pulse contains about $n$ peaks. In the above we argued that action-angle can not be introduced for our boundary model because $I_{3}^{\text {out }}$ is an essentially singular function of the initial parameters $\omega$ and $C$.

\section{Conclusions}

We considered a toy model of dilaton gravity with a dynamical boundary. As opposed to the original CGHS model, gravitational interactions in our model are weak in the Minkowski vacuum. The classical dynamics of this model in described by the single differential equation with an infinite number of exact solutions. The latter solutions describe either reflection of matter from the boundary or collapse. An important feature of the model is an existence of a minimal black hole mass $M_{c r}$. We studied the class of critical solutions separating regimes of total reflection and black hole formation.

We argued that despite an infinite number of exact solutions, our model is globally non-integrable. Namely, the natural conserved integrals in the outgoing and ingoing sectors cannot be smoothly glued at the boundary: the procedure fails in the vicinity of critical solution where the map of the integrals becomes singular.

Our two-dimensional model with dynamical boundary might be a useful tool for future researches of the semiclassical gravity.

\section{Acknowledgments}

We thank Sergey Sibiryakov for useful discussion. The work is supported by the RFBR grant 14-0231768 .

\section{References}

[1] S. W. Hawking, "Breakdown of Predictability in Gravitational Collapse," Phys. Rev. D 14 (1976) 2460.

[2] J. M. Maldacena, “Eternal black holes in anti-de Sitter,” JHEP 0304 (2003) 021.

[3] G. 't Hooft, "The black hole interpretation of string theory," Nucl. Phys. B 335 (1990) 138.

[4] J. Polchinski, "Chaos in the black hole S-matrix," arXiv:1505.08108 [hep-th].

[5] M. Fitkevich and D. Levkov, in preparation (2016).

[6] C. G. Callan, Jr., S. B. Giddings, J. A. Harvey and A. Strominger, "Evanescent black holes," Phys. Rev. D 45 (1992) R1005.

[7] D. Grumiller, W. Kummer and D. V. Vassilevich, "Dilaton gravity in two-dimensions," Phys. Rept. 369 (2002) 327.

[8] T. D. Chung and H. L. Verlinde, "Dynamical moving mirrors and black holes," Nucl. Phys. B 418 (1994) 305. 